University of Wollongong

Research Online

Faculty of Engineering - Papers (Archive)

Faculty of Engineering and Information

Sciences

June 1998

\title{
Nonlinear single-electron tunneling through individually coated colloid particles at room temperature
}

\author{
S. T. Yau \\ University of Wollongong \\ P. Mulvaney \\ University of Melbourne \\ W. Xu \\ University of Wollongong \\ Geoffrey M. Spinks \\ University of Wollongong, gspinks@uow.edu.au
}

Follow this and additional works at: https://ro.uow.edu.au/engpapers

Part of the Engineering Commons

https://ro.uow.edu.au/engpapers/248

\section{Recommended Citation}

Yau, S. T.; Mulvaney, P.; Xu, W.; and Spinks, Geoffrey M.: Nonlinear single-electron tunneling through individually coated colloid particles at room temperature 1998.

https://ro.uow.edu.au/engpapers/248

Research Online is the open access institutional repository for the University of Wollongong. For further information contact the UOW Library: research-pubs@uow.edu.au 


\title{
Nonlinear single-electron tunneling through individually coated colloid particles at room temperature
}

\author{
S.-T. Yau* \\ Department of Materials Engineering, University of Wollongong, NSW 2522, Australia \\ P. Mulvaney \\ School of Chemistry, The University of Melbourne, VIC 3052, Australia \\ W. Xu \\ Department of Physics, University of Wollongong, NSW 2522, Australia \\ G. M. Spinks \\ Department of Materials Engineering, University of Wollongong, NSW 2522, Australia
}

(Received 20 October 1997)

\begin{abstract}
Single-electron tunneling (SET) has been observed with nanometer coated colloid gold particles at room temperature. We have made the smallest (3-nm) thiol- and silicon dioxide $\left(\mathrm{SiO}_{2}\right)$-coated gold particles, from which we obtained SET signals using a scanning tunneling microscope (STM). STM images reveal individual particles supported by an atomically flat metal surface. The STM tip is used to obtain SET signals from the individual particles, whose shapes have been characterized. The current-voltage curves of the particles exhibit well-defined Coulomb staircases that resemble those obtained at $4.2 \mathrm{~K}$, indicating a strong Coulomb repulsive interaction at room temperature. The clear Coulomb staircases are due to a nonlinearity in the current steps. We suggest a possible mechanism for the nonlinearity in terms of many-body excitations in the particle. We have also identified the region of the particles, where the SET signal originates, using current-imaging-tunneling spectroscopy. We describe the advantages of using the coated nanometer particles for making devices for room-temperature operations. [S0163-1829(98)50924-1]
\end{abstract}

Nanometer metallic particles have physical properties different from those of the bulk. They are of technological importance since they can be used as "building blocks" to assemble novel materials and devices. At the nanometer scale, quantum mechanics gives rise to interesting phenomena such as single-electron tunneling ${ }^{1}$ (SET) and the quantum-size effect, which causes splitting in the otherwise continuous electron energy spectrum of the particles. ${ }^{2}$ In this paper, we report on the observation of SET in nanometer coated metal colloid particles. We also describe the preparation of the smallest silicon dioxide $\left(\mathrm{SiO}_{2}\right)$-coated $\left(\mathrm{Au} @ \mathrm{SiO}_{2}\right)$ and thiol-coated gold colloid particles. The work reported here is an experiment in which the measured SET signals are unambiguously shown to be associated with a particular single particle. We have also identified the region on the particle where the SET signals originate. Nonlinear processes occurring in the electron transport give rise to well-defined SET signals at room temperature. A possible explanation for the nonlinearity in terms of many-body excitation is given. Thus, our SET signals are suitable for roomtemperature device operations.

SET is an electron-transport process that occurs in the serial connection of two tunneling junctions, in which the number of excess electrons residing on the central electrode can be varied by varying the biasing voltage across the two outer electrodes. ${ }^{3,4}$ If the junction charging energy exceeds thermal fluctuations, there exists a narrow range about the zero-bias point, within which the tunneling current is suppressed. This is the so-called Coulomb blockade. If the bias is increased beyond the Coulomb blockade region, electrons start tunneling across the junctions in a highly correlated manner, which results in a series of current steps in the current-voltage $(I-V)$ curve. Each step indicates that the number of excess electrons on the central electrode $\Delta Q$ has increased by 1 . The series of current steps is called the Coulomb staircase.

Our method of preparing 3-nm $\mathrm{Au} @ \mathrm{SiO}_{2}$ and thiolcoated gold colloid particles is based on our previous results for the $15-\mathrm{nm}$ particles. ${ }^{5,6}$ The thiol-derivatized particles were prepared by adding $25 \mathrm{ml}$ of a solution containing sodium citrate $(15 \mathrm{ml}, 0.25 \%)$, tannic acid $(5 \mathrm{ml}, 1 \%)$, and $\mathrm{K}_{2} \mathrm{CO}_{3}\left(5 \mathrm{ml}, 0.01 \mathrm{~mol}\right.$ at $60{ }^{\circ} \mathrm{C}$ to a solution of $\mathrm{HAuCl}_{4}(85$ $\mathrm{ml}, 0.1 \%$ ) heated to $60^{\circ} \mathrm{C}$ and stirred vigorously. The fresh gold sol is stabilized by the polymeric tannic acid. This was removed by dialysis (final conductivity $<5 \mu \mathrm{S} \mathrm{cm}^{-1}$ ). Then 10- $\mu \mathrm{M}$ mercaptopropionic acid was added to the sol. It was then redialyzed to remove noncomplexed thiol. The final $\mathrm{pH}$ was 5.5. Tannic acid was found to interfere with silica deposition. To create 3-nm $\mathrm{Au} @ \mathrm{SiO}_{2}$ metal-insulator core-shell structures, a different protocol was adopted. $\mathrm{HAuCl}_{4}$ (5 $\left.\times 10^{-4} \mathrm{~mol}\right)$ was added to sodium citrate $(5 \mathrm{mM})$ at room temperature. To $100 \mathrm{ml}$ of the rapidly stirred solution, $100 \mu \mathrm{l}$ of ice-cold $\mathrm{NaBH}_{4}$ was added by pipette. The resultant orange sol had a surface plasmon resonance at $518 \mathrm{~nm}$. The particle size was $3 \mathrm{~nm}$ determined by TEM, with a standard deviation of just $12 \%$. A homogeneous silica coating was achieved by adding $0.5-\mathrm{ml}, 1-\mathrm{mM}$ (3-aminopropyl) trimethoxysilane to the gold sol at $p \mathrm{H} 5$. After $15 \mathrm{~min}$, com- 


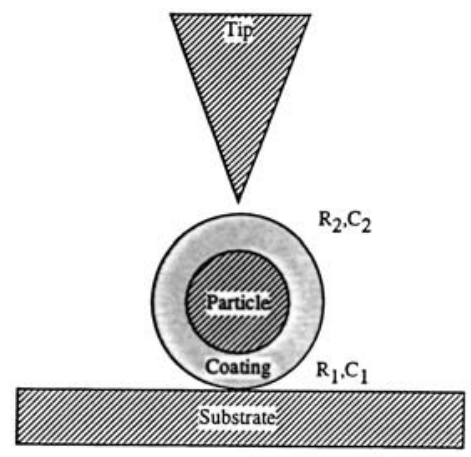

FIG. 1. A schematic description of the double tunneling junction system, showing the resistance and the capacitance of each individual junction. The total resistance $R$ is $R_{1}+R_{2}$.

plete complexation of amino groups with surface gold atoms takes place. A solution of active silica $(4 \mathrm{ml}, 0.54 \mathrm{wt} \%$ ion exchanged) is added to the surface modified gold sol. The resultant dispersion is aged at $p \mathrm{H} 9$ for $24 \mathrm{~h}$, at which point the shell is about 2-4 nm thick. Ethanol was then added to ensure final quantitative precipitation of silicate ions onto the surface.

Our double tunneling junction structure was formed by positioning the PtIr scanning tip of a scanning tunneling microscope (STM) above a colloid particle, which was deposited on a metal substrate as depicted in Fig. 1. To ensure that our data are associated with a single individual particle, we first used the STM to image this particle to reveal its shape and to show that the particle is isolated from others. Subsequently, we obtained $I-V$ curves of this particle with the STM tip positioned above the particle. The concentration of the colloid suspension can be adjusted so that deposition of the colloid forms either a thin film of particles or isolated single particles on the substrate surface. The substrate was prepared by sputtering gold onto a cleaved mica substrate. Annealing the gold substrate in a Bunsen flame results in flat regions that are $100 \mathrm{~nm}$ in dimension with $0.2-\mathrm{nm}$ steps. This is very crucial to the characterization of nanometer particles. The flat regions are in the shape of triangles and circular terraces, which belong to the $\mathrm{Au}(111)$ surface. $^{7}$

The STM images of a $\mathrm{Au} @ \mathrm{SiO}_{2}$ particle and a thiolcoated particle are shown in Figs. 2(a) and 2(c), respectively. Figures 2(b) and 2(d) show the $I-V$ curves of the particles. Each curve is the average of ten sweeps taken at the center of the particle, and the features are reproducible for each kind of particle. The Au@ $\mathrm{SiO}_{2}$ particle pointed to by the arrow has a diameter of $9 \mathrm{~nm}$. In its $I-V$ curve a series of current steps in both polarities away from the zero-bias region is clearly resolved. The seemingly featureless region near zero bias is due to the fact that the experiment was performed at an elevated temperature. ${ }^{3}$ However, the conductance $(d I / d V$ vs $V$ ) curve of Fig. 2(b) reveals the features in this Coulomb blockade region. The inset of Fig. 2(b) is a plot of conductance peak position versus peak index. Fitting to the plot yields a voltage-step width $\Delta V$ of $70 \mathrm{mV}$. The value of $\Delta V$ implies that $C_{L}$, which is the larger of $C_{1}$ and $C_{2}$, is 2.3 $\times 10^{18} \mathrm{~F}$, which satisifies the condition $e^{2} / 2 C_{L}>k_{B} T$ for the observation of the Coulomb staircase at room temperature. Figure 2(d) shows the $I-V$ and $d I / d V$ curves of the 11-nm thiol-coated gold particle shown in Fig. 2(c). From the value of $\Delta V, C_{L}$ is $1.7 \times 10^{-18} \mathrm{~F}$. The magnitude of the measured capacitances is of the same order as our calculated particle capacitances, ${ }^{8}$ while the exact values depend critically on the details of the junction geometry. Nevertheless, according to the orthodox theory, ${ }^{3,4}$ the presence of the equally spaced steps on the $I-V$ curves reflects the single-electron nature of the transport process. The inset of Fig. 2(d) is a calculated $I-V$ curve based on the method of Ref 9 . In the calculation, $R_{2}$ was set to be much larger than $R_{1}$ since it was observed that $R_{2}$ determines the slope of the $I$ - $V$ curves; $C_{1}$ was set to be equal to $C_{2}$, having the value of $1.7 \times 10^{-18} \mathrm{~F}$. The calculated curve reflects most of the features of the experimental curve. ${ }^{10}$ In particular, the smallest conductance peak, which causes asymmetry in both voltage and current in the $I-V$ curve, suggests that the fractional polarization charge on the particle, $Q_{0}$, is about $-0.48 e, e$ being the charge of an electron. The calculation also yields $Q_{0}=0.20 e$ for the $\mathrm{Au} @ \mathrm{SiO}_{2}$ particle of Fig. 2(b).

The steps of the Coulomb staircases in Figs. 2(b) and 2(d) are so clearly resolved that they can be compared to those obtained at $4.2 \mathrm{~K} .{ }^{11}$ The corners are rather sharp so that the distinct rise of the current step $\Delta I$ and the appearance of the plateau after the step is unambiguously displayed. The clear steps reflect the fact that the Coulomb repulsive interaction is strong enough at room temperature to keep $\Delta Q$ at the nominal value at the given bias. This is achieved at room temperature by having different transmission factors for the two junctions and small junction capacitances. These conditions were satisfied in our experiment with $R_{2} \gg R_{1}$ and very small capacitances as shown above. We point out that because the coating of the particle is uniform, which will be shown below, $R_{1}$ should be a constant. This is crucial for obtaining good and consistent SET signals as in our experiment. The Coulomb staircases show a disagreement with theory in that the current step $\Delta I$ increases with the magnitude of the bias $V$. This is an indication of nonlinear processes occurring in the transfer of electrons across the junctions. The nonlinearity could be due to the effects of density of state or a biasdependent tunneling matrix element (barrier effect). These effects to lowest order, will cause the slope of the envelope of the conductance-voltage curves to increase with the bias giving rise to the nonlinearity in $\Delta I$. However, by examining Figs. 2(b) and 2(d), we find a series of conductance oscillation with increasing amplitudes superimposed on a flat background. Thus, the nonlinearity occurring in our experiment is not due to density of state or barrier effect.

As an alternative, many-body excitations might be a possible explanation for the nonlinearity in $\Delta I$. As an excess electron just arrives at the particle, it appears as a hot electron whose energy $E$ is above the Fermi energy $E_{F}$ of the particle so that the electron distribution of the system is in nonequilibrium. Subsequent scattering events bring the hot electron to equilibrium, causing it to dissipate its excess energy by excitations of electron-hole $(e-h)$ pairs. For the size of the particles, the quantum-size effect is negligible so that at room temperature the particle energy spectrum is essentially continuous like that of the bulk material. We assume single-scattering events and perfect inelastic excitations, meaning that the hot electron undergoes only one scattering event during its stay on the particle and it loses the same amount of energy that the secondary electron gains. There- 
(a)
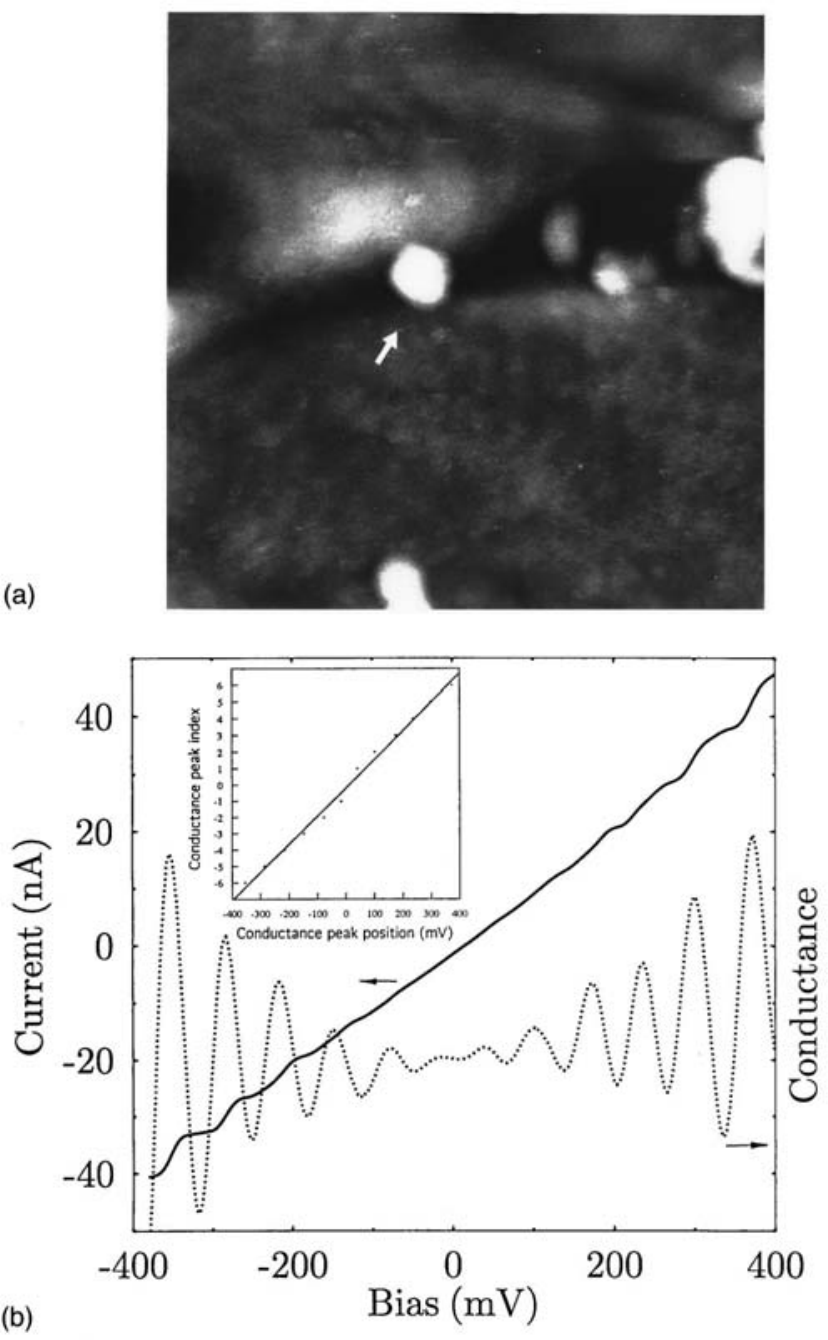

(c)
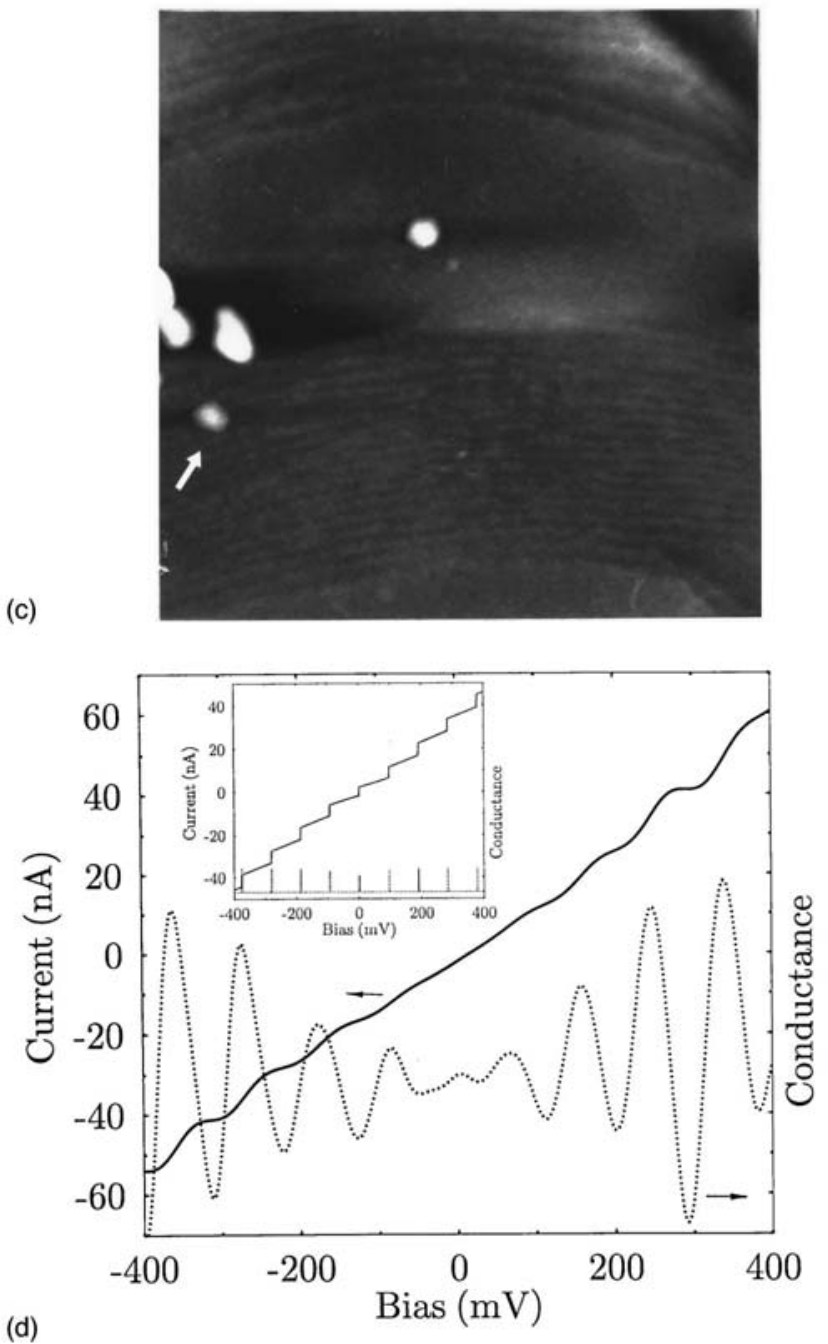

FIG. 2. (a) STM image $\left(100 \times 100 \mathrm{~nm}^{2}\right)$ of several $\mathrm{Au} @ \mathrm{SiO}_{2}$ particles deposited on an $\mathrm{Au}(111)$ surface; (b) $I-V$ curve of the particle pointed to by the arrow in (a). Also shown is the conductance curve ( $d I / d V$ vs $V$ ) obtained numerically. The inset is the plot of conductance peak index vs peak position; (c) STM image $\left(250 \times 250 \mathrm{~nm}^{2}\right)$ of several thiol-coated gold particles deposited on an Au(111) surface; (d) $I-V$ curve of the particle pointed to by the arrow in (c). Also shown is the conductance curve $(d I / d V$ vs $V)$ obtained numerically. The $I$ - $V$ curve shows a $R$ of $8.9 \mathrm{M} \Omega$. The inset shows the calculated $I-V$ vs $V$ (solid) and $d I / d V$ vs $V$ (dotted) curves, which are obtained with $C_{1}=C_{2}$ $=1.7 \times 10^{-18} \mathrm{~F}, R_{1}=0.4 \mathrm{M} \Omega, R_{2}=8.5 \mathrm{M} \Omega$, and $\mathrm{T}=300 \mathrm{~K}$.

fore, when a scattering event takes place on the metal particle, conservation of energy and momentum results in an electron below $E_{F}$ being excited above $E_{F}$ by the hot electron. This process generates a hole below $E_{F}$. The time for the hot electron to dissipate its energy and thus excite another electron $\tau_{r}$ is in our case on the order of $10^{-12} \mathrm{~s} .^{12}$ The dwell time $\tau_{d}$ during which the excess electron stay on the particle is calculated to be on the order of $10^{-11} \mathrm{~s}$. Thus, the excitations are likely to occur. Since the screening length in the metallic particle is extremely short $(\sim 1 \mathrm{~A})$, the excited secondary carriers (the electron and the hole) move independently before they participate in the current flow. Such current caused by secondary carriers has also been observed in the ballistic-electron-emission-microscopy-related scattering spectroscopy. ${ }^{13}$ The relaxation time of the hot electron $\tau_{r}$ has been calculated. ${ }^{14}$ In this work, the electron-hole-pairassisted tunneling in a nanojunction between a STM tip and a metallic substrate is treated. The generation of electron- hole pairs due to energy dissipation of hot electrons is shown to have a characteristic time of

$$
\tau_{r} \sim\left(E-E_{F}\right)^{2}
$$

This result has also been obtained in the treatment of bulk metals. ${ }^{15}$ Therefore, depending on the amount of energy the hot electron loses, the secondary carriers may have a $\tau_{r}$ that is greater than $\tau_{d}$. These secondary electrons will participate in the tunneling process, contributing to the current. The nonlinearity in $\Delta I$ is due to the fact that the number of hot electrons entering the particle increases by 1 each time the bias passes one step of the staircase and the fact that $\tau_{r}$ decreases as the square of the excess energy and hence the probability of generating secondary electrons becomes larger. Note that multiple-scattering events are also possible, and these processes also give rise to nonlinearity. The $I-V$ 


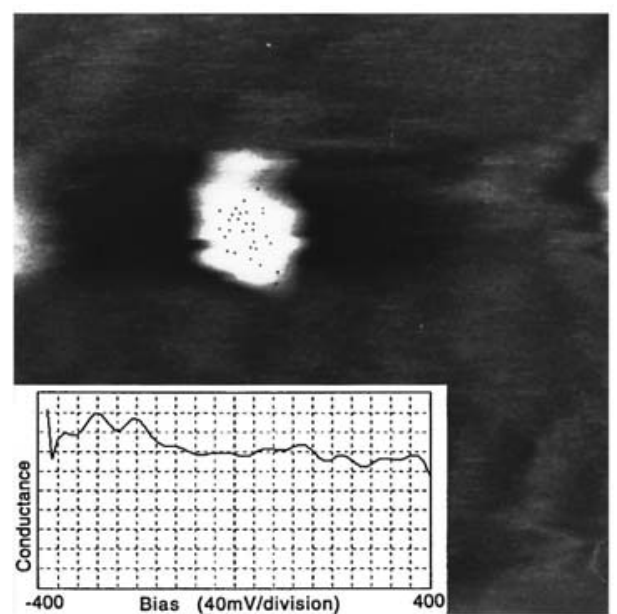

FIG. 3. CITS imaging $\left(50 \times 50 \mathrm{~nm}^{2}\right)$ of an $\mathrm{Au} @ \mathrm{SiO}_{2}$ particle deposited on an $\mathrm{Au}(111)$ surface. The black dots indicate the locations at which the $d I / d V$ vs $V$ curve shows equally spaced peaks such as those shown in the inset.

curves in Figs. 2(b) and 2(d) show that the nonlinear dependence of $\Delta I$ on $V$ in part gives rise to the clear current steps.

STM imaging does not reveal the shape of the gold particle inside the coating, which is important in the characterization of individual as-deposited colloid particles. It is also important to visualize the spatial distribution of the SET signal on the particle. For these reasons, we have performed current-imaging-tunneling spectroscopy (CITS), in which the STM makes an $I-V$ curve at each imaging point. Figure 3 shows a CITS image of a 9-nm $\mathrm{Au} @ \mathrm{SiO}_{2}$ particle. The black dots appearing on the particle indicate the locations where the $d I / d V$ curves show a series of equally spaced peaks such as the ones shown in the inset of Fig. 3. The SET signal is distributed approximately within a 3-nm circle about the center of the coated particle. Thus, we are certain that the gold particle indeed has a diameter of $3 \mathrm{~nm}$ and that the coating is uniform. Also, CITS ensures that the SET signal originates from the particle, not the coating or the substrate.

Our method of obtaining SET signals with the colloid particle shows several advantages. In recent work on roomtemperature operation of silicon SET devices, ${ }^{16-18}$ singleelectron transport is not obvious but is only estimated by estimating capacitances. It is very likely that the number of excess electrons stored on the devices exceeds the nominal value. In our case, the room-temperature Coulomb staircase shows well-defined equally spaced steps, indicating that one electron generates a usable signal for room-temperature operation. Also, low-bias (fractions of a V) operations reduce oxide degradation. As for device fabrication, when a colloid suspension is in contact with a solid substrate, the particles can be "coaxed" into nanometer patterns that have already been fabricated on the surface of the substrate. ${ }^{19}$ The particles can also form two-dimensional ordered monolayers. 5,6 Therefore, colloid particles can be used as building blocks of complex metal-insulator systems, such as the SET devices, in which several tunneling junctions (coated particles) are connected to sustain solitons. With the $\mathrm{SiO}_{2}$ and thiol coatings, colloid particles allow fabrication of planar multiple tunneling junctions with fewer processing steps compared to the silicon technology. Also, both the metal core size and the thickness of the coating can be readily controlled, ${ }^{6}$ allowing both resistances and capacitances to be tuned. Thus, our method can either be incorporated into the silicon processing technology or be used to develop a metal-based device fabrication. Finally, we emphasize that many aspects of our results have to be studied in detail.

Helpful discussions with C. Zhang are gratefully acknowledged.
*Electronic address: zyausiu@ @resto.net.au

${ }^{1}$ For a general review of single-electron tunneling, see M. H. Devoret et al., Nature (London) 360, 547 (1992); K. K. Likharev and T. Cleason, Sci. Am. (Int. Ed.) 6, 50 (1992).

${ }^{2}$ R. Kubo, J. Phys. Soc. Jpn. 17, 975 (1962); D. C. Ralph, C. T. Black, and M. Tinkham, Phys. Rev. Lett. 74, 3241 (1995).

${ }^{3}$ K. K. Likharev, IEEE Trans. Magn. 23, 1142 (1987).

${ }^{4}$ K. Mullen, Phys. Rev. B 37, 98 (1988).

${ }^{5}$ M. Giersig and P. Mulvaney, Langmuir 9, 3408 (1993),

${ }^{6}$ L. M. Liz-Marzan, M. Giersig, and P. Mulvaney, Langmuir 12, 4329 (1996).

${ }^{7}$ Z. Wang and M. Moskovits, J. Appl. Phys. 71, 5401 (1992).

${ }^{8}$ We calculate the capacitances using B. Laikhtman and E. L. Wolf, Phys. Lett. A 139, 257 (1989), Eq. (8).

${ }^{9}$ A. E. Hanna and M. Tinkham, Phys. Rev. B 44, 5919 (1991).
${ }^{10}$ The calculation does not include the effect of finite temperature, which smooths the curve.

${ }^{11}$ R. Wilkins, E. Ben-Jacob, and R. C. Jaklevic, Phys. Rev. Lett. 63, 801 (1989).

12 N. W. Ashcroft, and N. D. Mernin, Solid State Physics (Saunders College, Philadelphia, 1976).

${ }^{13}$ L. D. Bell, M. H. Hecht, W. J. Kaiser, and L. C. Davis, Phys. Rev. Lett. 64, 2679 (1990).

${ }^{14}$ F. Flores, P. M. Echenique, and R. H. Ritchie, Phys. Rev. B 34, 2899 (1986).

${ }^{15}$ J. J. Quinn and F. A. Ferrell, Phys. Rev. 122, 812 (1958).

${ }^{16}$ K. Yano et al., IEEE Trans. Electron Devices 41, 1628 (1994).

${ }^{17}$ S. Tiwari et al., Appl. Phys. Lett. 68, 1377 (1996).

${ }^{18}$ L. Guo, E. Leobandung, and Y. S. Chou, Science 275, 649 (1997).

${ }^{19}$ S.-T. Yau and Y. Zhou, Mod. Phys. Lett. B 9, 187 (1995). 